Proc. of the 11 Int. School on Theoretical Physics Symmetry and Structural Properties of Condensed Matter, Rzeszów 2014

\title{
Graphene Conductance
}

\section{in the Presence of Resonant Impurities}

\author{
M. INGLOT ${ }^{a, *}$ AND V.K. DUGAEV ${ }^{b, c}$ \\ ${ }^{a}$ Research and Development Centre for Photovoltaics, ML System Sp. z o.o., \\ Warszawska 50D, 35-230 Rzeszów, Poland \\ ${ }^{b}$ Department of Physics, Rzeszów University of Technology, al. Powstańców Warszawy 6, 35-959 Rzeszów, Poland \\ ${ }^{c}$ Departamento de Física and CeFEMA, Instituto Superior Técnico, Universidade de Lisboa, \\ av. Rovisco Pais, 1049-001 Lisbon, Portugal
}

\begin{abstract}
We discuss transport properties of graphene related to the resonant scattering from impurities and defects. Two different models describing defects in the bulk of graphene or at the graphene surface are used for the calculation of self energy of electrons scattered from short-range impurities or defects. The results of numerical calculations demonstrate a resonant character of resistance. In the case of neutral impurities or defects the scattering also leads to a resonant decrease of the spin relaxation time.
\end{abstract}

DOI: $10.12693 /$ APhysPolA.128.163

PACS: 72.10.Fk, 72.80.Vp, 73.20.Hb

\section{Introduction}

Transport properties of graphene are affected by impurities and defects, which can be located at the graphene surface, flake edges, or incorporated in the crystal lattice substituting the carbon atoms [1-3]. Besides, there is always some density of vacancies created thermodynamically or generated by irradiation. Like in the case of conventional semiconductors, the impurity perturbation can be responsible for creation of impurity states in graphene. However, the relativistic energy spectrum of graphene $[1,2,4]$ makes this problem quite different from the usual semiconductor $[1,5]$. One of such pecularities is related to the absence of gap, namely, if the impurity state is formed it should be quasilocalized, which correponds to the resonance in the density of states.

In this work we consider the case of strongly localized perturbation located within the lattice of graphene or at the surface. In the latter case, the mirror symmetry is broken locally, leading to the possibility of localized Rashba spin-orbit perturbation [6]. As a result, scattering from the adatoms at the graphene surface can determine both the momentum and spin relaxation times.

The model with short-range perturbation potential correponds to a neutral impurity or defect. This model has been used for graphene $[7,8]$ to determine the possible effect on its transport [7] and thermoelectric properties $[9,10]$.

\section{Model}

The electronic structure of graphene in the vicinity of Dirac points $K$ and $K^{\prime}$ can be described by the following Hamiltonian [11]:

\footnotetext{
* corresponding author; e-mail: miinglot@gmail.com
}

$$
\hat{H}_{0}=\hbar v\left( \pm \tau_{x} k_{x}+\tau_{y} k_{y}\right),
$$

where $v=c / 300$ is the electron velocity in graphene, $c$ is the speed of light, $\tau_{x}$ and $\tau_{y}$ are the Pauli matrices operating in sublattice (pseudospin) space. The plus/minus sign in Eq. (1) refers to the electrons in non-equivalent $K$ and $K^{\prime}$ Dirac points, respectively.

The Hamiltonian of Eq. (1) can be diagonalized by a unitary transformation and gives the valence and conduction bands with linear dispersion $\varepsilon_{1,2}(\boldsymbol{k})= \pm \hbar v k$.

The electric current $j$ in graphene can be induced by the in-plane electric field $E$ and/or by the temperature gradient $\nabla T$. To find the distribution function of electrons in the $n$-th energy band, $f^{(n)}(\boldsymbol{k})$, of the system in non-equilibrium we use the Boltzmann kinetic equation. In the case of small deviation from equilibrium we get

$$
-v_{x}^{(n)} \frac{\partial f_{0}}{\partial \varepsilon}\left(\nabla \mu+\frac{\varepsilon-\mu}{T} \nabla T-e E\right)=-\frac{\delta f^{(n)}}{\tau_{n}},
$$

where $\delta f^{(n)}(\boldsymbol{k})$ is the deviation from the Fermi-Dirac distribution function $f_{0}(\varepsilon), \tau_{n}(\varepsilon)$ is the electron relaxation time, and $v_{x}^{(n)}$ is the $x$-component of electron velocity, $\boldsymbol{v}^{(1,2)}(\boldsymbol{k})= \pm v \boldsymbol{k} / k$. For definiteness, we take the electric field and temperature gradient along axis $x$.

In a general case, when the current density $j$ is induced by the field $E$, temperature gradient $\nabla T$, and gradient of chemical potential $\nabla \mu$, we write

$$
\begin{aligned}
& j=e \sum_{n \boldsymbol{k}} v_{x}^{(n)} \delta f^{(n)}= \\
& e^{2} K_{11} E-e K_{11} T \nabla \frac{\mu}{T}-e K_{21} \frac{\nabla T}{T},
\end{aligned}
$$

where $K_{r s}$ are the kinetic coefficients [12].

Using the linearity of the energy spectrum near $K$ and $K^{\prime}$ Dirac points, one can find the kinetic coefficients for graphene 


$$
K_{r s}=-\frac{1}{4 \pi \hbar^{2}} \sum_{n} \int_{-\infty}^{\infty}|\varepsilon| \varepsilon^{r-1} \tau_{n}^{s}(\varepsilon) \frac{\partial f_{0}}{\partial \varepsilon} \mathrm{d} \varepsilon .
$$

This expression includes the contribution of valence and conduction bands.

The electric conductivity is related to $K_{11}$ :

$$
\sigma=e^{2} K_{11} \text {. }
$$

As follows from Eqs. (4) and (5), the dependence of relaxation time on energy is especially important for the conductivity.

\section{Relaxation time}

The graphene sheets can be doped with impurities in two different ways. Namely, the perturbation potential can be located within the plane (e.g., impurity is replacing the carbon atom), or it can be located at one side of the graphene surface (impurity adatom). In the first case we can take for the single-impurity perturbation $\hat{V}_{1}^{(A, B)}=V_{1}^{(A, B)} \delta\left(\boldsymbol{r}-\boldsymbol{R}^{(A, B)}\right)$, where $A, B$ denotes graphene sublattice, which is occupied by impurity. In the second case, one can identify such perturbation as a local Rashba spin-orbit coupling in graphene, which has the form $\hat{V}_{2}=V_{2} \delta(\boldsymbol{r})\left(\tau_{x} \sigma_{y}-\tau_{y} \sigma_{x}\right)$.

The Hamiltonian which includes any kind of impurityinduced perturbation is $\hat{H}=\hat{H}_{0}+\sum_{i} \hat{V}_{m}\left(\boldsymbol{r}-\boldsymbol{R}_{i}\right)$, where the sum runs over all randomly distributed impurities located at $\boldsymbol{R}_{i}$ positions, and $m=1,2$ refers to a chosen model. Note that the model assumes a short-range randomly distributed impurity potential with equal probability in the sublattice $A$ and $B$ (for intrinsic impurity), and a homogeneous distribution above or below graphene sheet (extrinsic impurity).

Using the $T$-matrix approach we can find the resonant energy level in graphene. It corresponds to the impurity scattering in all orders of perturbation. The equation for the $T$-matrix is

$$
\hat{T}_{\boldsymbol{k} \boldsymbol{k}^{\prime}}(\varepsilon)=\hat{V}_{\boldsymbol{k} \boldsymbol{k}^{\prime}}+\sum_{\boldsymbol{k}^{\prime \prime}} \hat{V}_{\boldsymbol{k} \boldsymbol{k}^{\prime \prime}} \hat{G}_{\boldsymbol{k}^{\prime \prime}}(\varepsilon) \hat{T}_{\boldsymbol{k}^{\prime \prime} \boldsymbol{k}^{\prime}}
$$

where $\hat{G}_{\boldsymbol{k}}(\varepsilon)$ is the electron Green function related to unperturbed Hamiltonian $\hat{H}_{0}$. For the short-ranged perturbation potential we find the $T$-matrix in the following form:

$$
\hat{T}_{m}(\varepsilon)=\left(1-\hat{V}_{m} F(\varepsilon)\right)^{-1} \hat{V}_{m},
$$

where $F(\varepsilon)$ is

$$
F(\varepsilon)=\sum_{k} \frac{\varepsilon}{(\varepsilon+\mathrm{i} \delta)^{2}-(\hbar v k)^{2}} .
$$

Using Eq. (8) we can find for both types of impurities an equation determining the resonance energy, $\theta_{m}(\varepsilon)=0$, where we denote $\theta_{1}(\varepsilon)=1-V_{1} F(\varepsilon)$ (for intrinsic impurities), and $\theta_{2}(\varepsilon)=1-4 V_{2}^{2} F^{2}(\varepsilon)$ (for extrinsic impurity).

To find the relaxation time in the $n$th energy band ( $n=1,2)$ we have to diagonalize the effective Hamiltonian $\hat{H}_{\text {eff }}=\hat{H}_{0}+\hat{\Sigma}_{m}(\varepsilon)$, where $\hat{\Sigma}_{m}(\varepsilon)=N_{i} \hat{T}_{m}(\varepsilon)$ is the self-energy related toresonanse impurity scattering. Then the relaxation time of an electrons in the $n$-th band can be found from

$$
\frac{\hbar}{\tau_{n}^{(m)}(\varepsilon)}=2 \operatorname{Im} \Sigma_{n}^{(m)}(\varepsilon),
$$

where $\Sigma_{n}^{(m)}(\varepsilon)$ is the self energy after diagonalization of $\hat{H}_{\text {eff }}$.

The real and imaginary parts of function $F(\varepsilon)$ can be calculated from Eq. (8):

$$
\begin{aligned}
& \operatorname{Re} F(\varepsilon) \simeq-\frac{\varepsilon}{2 \pi(\hbar v)^{2}} \ln \frac{\hbar v k_{\max }}{|\varepsilon|}, \\
& \operatorname{Im} F(\varepsilon) \simeq-\frac{\varepsilon}{4(\hbar v)^{2}} .
\end{aligned}
$$

Here $k_{\max }$ is the wave-vector cutoff for the linear dispersion in graphene, $k_{\max } \simeq(|\boldsymbol{K}|+|\boldsymbol{M}|) / 2$, with $\boldsymbol{K}$ and $\boldsymbol{M}$ denoting the wave vectors corresponding to the points $K$ and $M$ of the graphene Brillouin zone.

The resonant levels in the case of intrinsic perturbation correspond to negative energy for positive impurity potential $V_{1}>0$, whereas for $V_{1}<0$ the energy of resonance is positive. In the case of extrinsic perturbations, the sign of impurity potential is not relevant, and the resonant states are located symmetrically at $\pm \varepsilon$. The resonance level $\varepsilon$ strongly depends on the strength of impurity potential $V_{1,2}$, and this is reflected in the momentum relaxation time $\tau_{n}(\varepsilon)$. Note that the relaxation time becomes divergent in the Dirac points, $\varepsilon=0$.

\section{Numerical results}

In the presence of resonant impurity, by using Eqs. (4), (5), and (9) we calculated the conductivity $\sigma$. As we see in Figs. 1 and 2, the conductivity strongly depends on the position of chemical potential $\mu$.
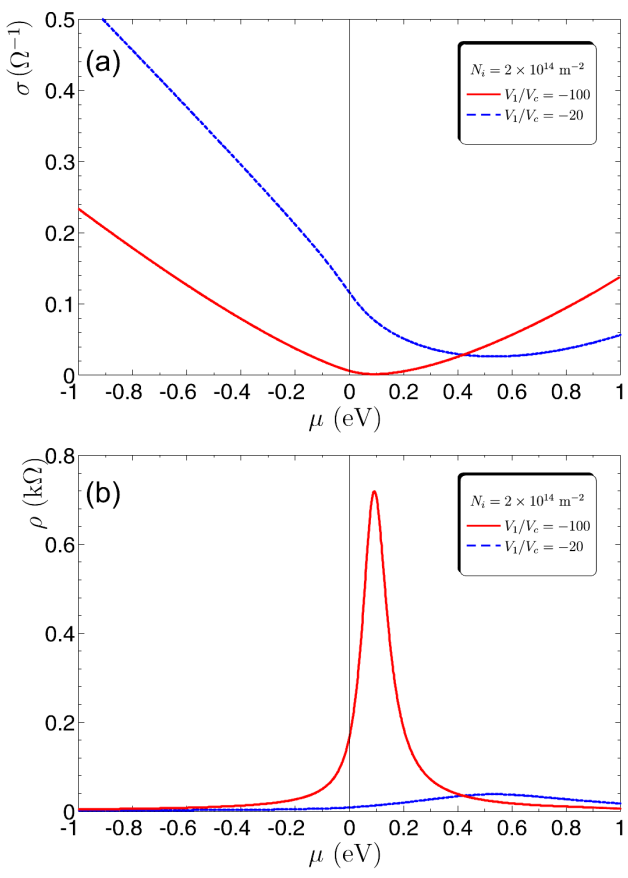

Fig. 1. (a) Electrical conductivity $\sigma$ and (b) resistivity $\rho$ as function of chemical potential $\mu$. Here the impurity concentration is $N_{i}=2 \times 10^{14} \mathrm{~m}^{-2}$, and temperature $T=300 \mathrm{~K}$. 

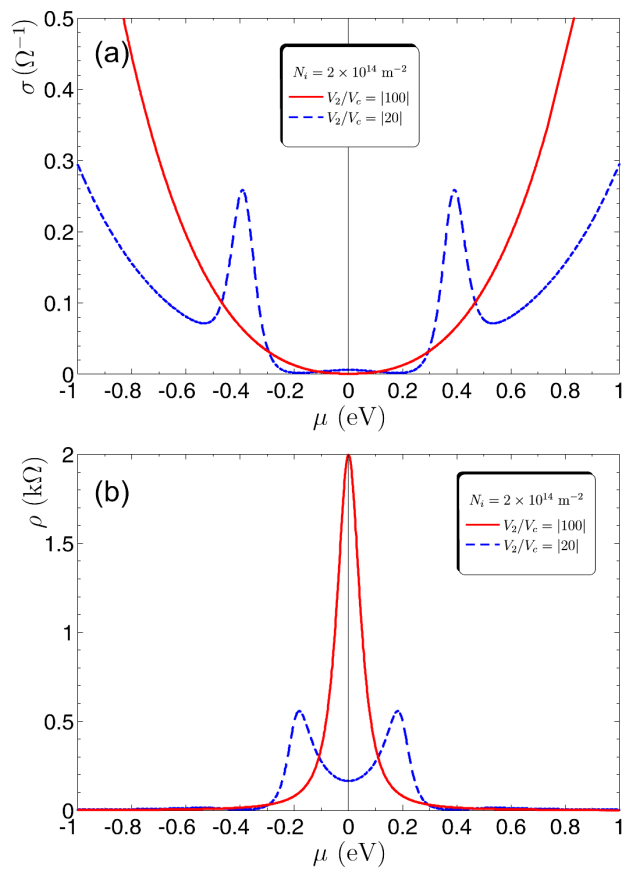

Fig. 2. (a) Conductivity and (b) resistivity of graphene vs. chemical potential $\mu$ for chosen impurity potential $V_{0}$ in the case of disordered Rashba SO perturbation. In both figures the impurity concentration $N_{i}=2 \times 10^{14} \mathrm{~m}^{-2}$, and the temperature $T=300 \mathrm{~K}$.

Figure 1a shows the dependence of conductivity $\sigma$ on the chemical potential $\mu$ for different impurity potentials, $V_{1}<0$. In the region of $|\mu|<1 \mathrm{eV}$ the conductivity monotonically decreases and has a minimum for chemical potential equal to the energy of localized state. For strong impurity potential $V_{1} \rightarrow \pm \infty$ (like for the graphene vacancy), the conductivity $\sigma$ goes to zero. The minimum of the curve is related to the position of resonant level. The resistance for the corresponding chemical potential has a strong maximum.

Scattering from the localized Rashba spin-orbit coupling perturbation is also affecting the spin relaxation time because electrons are spin-flip scatterred. Therefore, the resonant behavior of the momentum relaxation time can be also viewed as a resonance (minimum) in the electron spin relaxation.

\section{Conclusions}

We calculated the momentum relaxation time of electrons in graphene scattered from randomly distributed short range potentials related to impurities and defects. The calculation includes all orders of perturbation, which leads to appearance of the resonant states and, correspondingly, to strong decrease of the relaxation time of electrons with energy near the resonant state.

\section{Acknowledgments}

This work is supported by the projects Nos. POIG.01.04.00-18-101/12 and UDA-RPPK.01.03.00-18025/13-00 (ML System) and by the National Science Center in Poland under project Harmonia No. DEC2012/06/M/ST3/00042.

\section{References}

[1] M.I. Katsnelson, Graphene: Carbon in Two Dimensions, Camridge University Press, Cambridge 2012.

[2] A.H. Castro Neto, F. Guinea, N.M.R. Peres, K.S. Novoselov, A.K. Geim, Rev. Mod. Phys. 81, 109 (2009).

[3] N.M.R. Peres, Rev. Mod. Phys. 82, 2673 (2010).

[4] A.K. Geim, K.S. Novoselov, Nature Mater. 6, 183 (2007).

[5] A.V. Shytov, M.I. Katsnelson, L.S. Levitov, Phys. Rev. Lett. 99, 236801 (2007); Phys. Rev. Lett. 99 246802 (2007).

[6] D.V. Fedorov, M. Gradhand, S. Ostanin, I.V. Maznichenko, A. Ernst, J. Fabian, I. Mertig, Phys. Rev. Lett. 110, 156602 (2013).

[7] N.M.R. Peres, F. Guinea, A.H. Castro Neto, Phys. Rev. B 73, 125411 (2005).

[8] M. Inglot, V.K. Dugaev, J. Appl. Phys. 109, 123709 (2011).

[9] T. Löfwander, M. Fogelstrom, Phys. Rev. B 76, 193401 (2007).

[10] M. Inglot, A. Dyrdal, V.K. Dugaev, J. Barnaś, Phys. Rev. B 91, 115410 (2015).

[11] C.L. Kane, E.J. Mele, Phys. Rev. Lett. 95, 226801 (2005).

[12] P.S. Kireev, Semiconductor Physics, Mir Publishers, Moscow 1978. 